\title{
Editorial
}

\section{Importance of Family Medicine: Bangladesh Perspective}

\author{
Md. Zakiur RAHMAN
}

\begin{abstract}
Professor, Department of Microbiology, Monno Medical College, Manikgonj, Bangladesh \& Faculty of Bangladesh College of General Practitioners \& Bangladesh Academy of Family Physicians \& Secretary, Spice Route Young Doctors Movement, Bangladesh and Direct Member, World Organization of National College \& Academics of Family Medicine (WONCA); Email: profzakiur@gmail.com
\end{abstract}

The Family Physicians provide care in the community for people and their families of all ages regardless of their sex or income. As the first port of call for virtually all health related problems, the Family physician is at front line of health service. This physician deals with $90 \%$ of all illness, both in the chamber and in patient's homes ${ }^{1}$.

The job definition of the Family Physician agreed by the Leeuwenhorst group in 1974 and accepted by many educational bodies as the best available definition to date: "The Family Physician is a licensed medical graduate who gives personal, primary and continuing care to individual, families and a practice population, irrespective of age, sex and illness. It is the synthesis of these functions, which is unique. The physician will attend the patients in consulting room and in their homes and sometimes in a clinic or hospital. The aim is to make early diagnoses. The physician will include and integrate physical, psychological and social factors in the considerations about health and illness. This will be expressed in the care of the patients. This physician will make an initial decision about every problem. Furthermore, this physician will undertake the continuing management of the patients with chronic recurrent or terminal illness. Prolonged contact means that the physician can use repeated opportunities to gather information at a pace appropriate to each patient and build up a relationship of trust, which he can use professionally.

The family physician will practice in co-operation with other colleagues, medical and non-medical. The physician will know how and when to intervene through treatment, prevention and education to promote the health of his patient and their families. He will recognize that he also has a professional responsibility to the community. The recognition of Family Medicine as a specialty in its own right developed simultaneously in the United Kingdom, in Europe and in whole World. Family
Medicine is a specialized discipline of medical practice just as Cardiology, Nephrology Gerontology and so on.

National Prof. N. Islam plays a pioneering key role for the establishment and development of Family Medicine in Bangladesh. Dr. Robert W. Higgins, Former President of WONCA, has recognized his work by saying- "The Contribution of National Prof. N. Islam in the field of Family Medicine not only will improve the health care in Bangladesh, but also will come to serve as a role model for many other nations who are searching for solutions to their health care problems."

The Bangladesh Institute of Family Medicine \& Research is an Institute of the University of Science $\&$ Technology, Chittagong (USTC). My vision for the USTC is to consolidate our position as an outstanding regional university with a national and international reputation for quality. It is proud to be at the leading edge of a number of high profile initiatives; Family Medicine Diploma is one of them. The one-year long 'Family Medicine Diploma [FMD]' course, originally run by the USTC Dhaka Campus, is run by the Institute. The Family Physicians provide care in the community for people and members of the family irrespective of their age or sex. As the first port of call, virtually all health related problems are tackled by the Family Physicians. The role of Family Physicians is now a priority issue in the context of Bangladesh. Welltrained Family Physicians at all levels by their active participation can contribute substantially in achieving the goal of Health for All' in Bangladesh. Family Medicine has no frontier, as there is constant adding of new things to it in response to everyday social needs. Family Medicine Diploma [FMD] course offers outstanding students the opportunity to make a significant and lasting contribution to the field of Family Medicine in Bangladesh. We are very much confident that, very 
soon we shall be able to introduce MD course in Family Medicine.

Family Physicians without proper training and experiences lead to improper diagnosis and management. The need for adequate education and training in Family Medicine exists both in developed countries and in developing countries like Bangladesh. The challenge of providing adequate training in Family Medicine is made more difficult by the diversity of the subject. The situation of Family Medicine Practitioners in Bangladesh is a bit different from that of other countries. The Family Physicians of Bangladesh need education and training, but it is very much difficult for a practitioner to spare his practice. So, considering the situation, the Bangladesh Institute of Family Medicine \& Research, the University of Science \& Technology Chittagong has chalked out a 2-Step education programme for the Family Physicians in Bangladesh.

The Directorate of Family Planning is concerned with birth control, maternal and child health while the Directorate General of Health Services is responsible for overall health care of the country. Under these two Departments 341 health complexes and 2329 health centres have been established in the country at the sub-district and union levels respectively, which are the two smallest administrative units in Bangladesh. The Union Health centres are staffed by 1-2 graduate physicians and a number of paramedics and traditional birth attendants (TBA), while 9 graduate physicians and several nurses, paramedics, TBAs, laboratory technicians and other support staff are posted in the sub-district health complexes. However in most cases, the more remote and peripheral sub-district health complexes and union health centres are under-staffed.

The physician: patient ratio in Bangladesh is 1:4775. Most physicians are based in urban areas meaning that the scenario is even poorer in the rural communities, where primary health care is provided by quacks, rural medical practitioners, traditional medicine practitioners and paramedics.
Only 30\% of the population of Bangladesh has access to primary health care. In the urban areas, in big cities, district towns and municipalities, people mainly depend on medical graduates, fresh or experienced, and specialists for their every day health needs. Specialists play an important role in providing primary health care in Bangladesh. No referral is needed to consult a specialist physician here. Patients usually consult the respective specialists based on their initial idea about their disease. So whereas a patient with complaints of headache resulting from a brain tumor may correctly go to a Neurosurgeon directly at the very beginning, more commonly they end up in the wrong consultation chambers. For example patients with irritable bowel syndrome often consult Hepatologists for fear of cirrhosis of liver.

Bangladeshi patients can access primary health care through different gateways, which adds a huge problem. Not only the patients suffer because of the huge difference in the quality and standard of health care they receive, they are also vulnerable to maltreatment in the hands of non-qualified practitioners like quacks, rural medical practitioners, traditional medical practitioners and so on. Besides, over the years, the situation has become so difficult and complex that it will take years to put primary health care on the right track in Bangladesh. We are trailing far behind what the Brazilians have achieved by establishing 'health teams' comprising GP, nurse, nurse assistants and community agents with the support of World Bank.

Primary health care in Bangladesh is financed by both the public and the private sectors. But the majority of the 140 million Bangladeshis use the public system. In 2005, Bangladesh utilized a significant portion of her gross national product in health care and a bulk of this money went to primary health care.

[Journal of Current and Advance Medical Research 2016;3(2):31-32] 